\title{
CONSTRUCCIONES DE ENLACE TAIL-HEAD EN QUICHUA SANTIAGUEÑO
}

\author{
Head-tail linkage constructions in quichua santiagueño
}

\author{
MAYRA JUANATEY \\ CONICET-Universidad de Buenos Aires (Argentina) \\ mayrajuanatey@gmail.com \\ MARTÍNCALIFA \\ CONICET-Universidad Nacional de General Sarmiento (Argentina) \\ mcalifa@campus.ungs.edu.ar
}

\section{Resumen}

En muchas lenguas las cláusulas adverbiales iniciales presentan una repetición del predicado de la oración anterior, lo que se conoce como enlace tail-head (tail-head linkage, ETH de aquí en más) (Thompson y Longacre, 1985; de Vries, 2005). Este trabajo busca describir las construcciones de ETH del quichua santiagueño (quechua) de acuerdo con dos parámetros: a) el grado de solapamiento semántico entre los predicados de la construcción de ETH, y b) el grado de integración eventiva de la adverbial inicial con la cláusula principal. El primer parámetro permite identificar construcciones verbatim -con repetición exacta del verbo anterior-y no verbatim -sin repetición exacta-. El segundo parámetro se manifiesta en la forma que adopta la cláusula "cabeza". Así, se identificaron, por un lado, una forma converbal y, por el otro, cláusulas con los sufijos del sistema de conmutación de la referencia. La interacción entre estos dos parámetros muestra que las diferentes construcciones de ETH poseen diversas funciones discursivas, en particular para la coherencia temática (Givón, 2001).

Palabras clave: Quichua santiagueño; enlaces head-tail; cláusulas adverbiales; coherencia temática.

\section{Abstract}

In many languages preposed adverbial clauses repeat the previous verb or predicate, which phenomenon has been termed tail-head linkage (THL henceforth, Thompson \& Longacre, 1985; de Vries, 2005). This paper aims to describe THL constructions in Santiagueño Quichua (Quechua) according to two parameters: a) the degree of semantic overlap between the predicates of the THL constructions, and b) the degree of event integration of the preposed adverbial with its main clause. The first parameter allows the identification of verbatim constructions -with exact repetition of the previous predicate- and non verbatim constructions -without exact repetition-. The second parameter manifests itself in the form the head clause might adopt, where it is a converb or it takes switch reference suffixes. The interplay of both parameters shows that the different THL constructions possess diverse discourse functions, particularly regarding thematic coherence (Givón, 2001).

Key words: Santiagueño Quichua; head-tail linkage; adverbial clauses; thematic coherence. 


\section{INTRODUCCIÓN}

Las lenguas del mundo disponen de múltiples y variados mecanismos para establecer conexiones en el discurso. Entre estos, en algunas de ellas se destaca la posibilidad de que una cláusula de naturaleza adverbial en posición inicial repita el contenido de una cláusula anterior. Esto se ilustra en (1) con un ejemplo del kombai (awyu-dumut, Papúa Nueva Guinea).

$$
\begin{aligned}
& \text { a Kha-negena refe } \\
& \text { ir.3SG.NF-hasta.DS año uno } \\
& \text { "Continuó por un año y luego murió". } \\
& \text { b. Khumolei-n-a ifamano. } \\
& \text { morir.3SG.NF-TR-DS enterrar.3PL.NF } \\
& \text { "Murió y lo enterraron". }
\end{aligned}
$$
$\begin{array}{ll}\text { büwene-n- } a & \underline{\text { khumolei }} \\ \text { terminado.3SG.NF-TR-DS } & \underline{\text { morir.3SG.NF }}\end{array}$

Aquí puede verse cómo la cláusula inicial de la oración (1.b) -la "cabeza" (head)replica el predicado de la cláusula final de la oración (1.a) -la "cola" (tail)-. Fenómenos de este tipo han sido denominados construcciones de enlace tail-head (tail-head linkage; ETH de aquí en más) (Thompson y Longacre, 1985; Thompson, Longacre y Hwang, 2007) o, alternativamente, construcciones de bridging (Dixon, 2009).

Documentadas en distintas partes del mundo, las construcciones de ETH han sido estudiadas en profundidad en las lenguas de las Filipinas (Longacre, 1968, citado en Thompson, Longacre y Hwang, 2007) y Papúa Nueva Guinea (de Vries, 2005). En Sudamérica hay trabajos que las documentan y describen en lenguas como el aguaruna (jíbara) (Overall, 2009), el yurakaré (aislada) (Van Gijn, 2014), el cavineña (pano) y en la familia arawak (Guillaume, 2011). A este diverso conjunto deben sumarse, además, lenguas de la familia quechua, como el inga de Colombia (Thompson, Longacre y Hwang, 1985) y los quechuas andinos (Adelaar y Muysken, 2004) y amazónicos (Juanatey, 2019).

El quichua santiagueño, hablado sobre todo en la provincia de Santiago del Estero, Argentina, es otro de los miembros de esta última familia que exhibe construcciones de ETH. Considérese el siguiente ejemplo:

$\begin{array}{llll}\text { (2) a. } \begin{array}{ll}\text { wañu-s } \\ \text { morir-CONV }\end{array} & \frac{\text { lloqse-ra- } \phi}{\text { salir-PAS-3SG }} \\ \text { "Murió y (su alma) salió de su casa". } & \text { wasi-n-manta } \\ & \text { casa-POS.3SG-ABL }\end{array}$ 


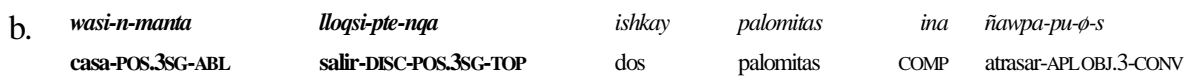

"Cuando salió de su casa dos palomitas le fueron detrás".

(Tebes y Karlovich, 2010, p. 214)

En (2) se observa que -al igual que en el ejemplo del kombai en (1)- la cláusula inicial de la segunda oración (2.b) repite el verbo lloqsera "salió" de la oración anterior (2.a). Nótese que, además del verbo, también se retoma el complemento wasinmanta "desde su casa".

En ocasiones, sin embargo, es posible observar que la cláusula inicial no constituye una repetición exacta de la anterior, como se ve en (3).

(3)
a.
lloqsi-sa
salir-NMIZ.ANT
ka-ra- $\phi$
COP-PAS-3SG
ri-s
ir-CONV
marcha-s
allim: itan-ta
"Salió cam
b. cha-yna
DEM.MED-COMP
ir-CONV encontrar-NMIZ.ANT
$k a-r a-\phi$
COP-PAS-3SG
$\begin{array}{ll}\text { suk } & \text { huell-ita-ta } \\ \text { una } & \text { huella-DIM-AC }\end{array}$
"Así yendo, encontró una huellita".
(Tevez en Albarracín, 2016)

En (3.b) se emplea la forma ris del verbo ri- "ir" para retomar lloqsisa kara "salió" de (3.a). La forma ris, además, aquí está acompañada por el demostrativo chayna "así/como eso". Las construcciones de ETH que, como en (3), no repiten exactamente el predicado anterior también han sido reconocidas en lenguas como el yurakaré (Van Gijn, 2014, pp. 295-98) y las lenguas papuanas wambon, kombai y korowai (de Vries, 2005, p. 376).

El objetivo de este trabajo, entonces, es describir y analizar las diferentes construcciones de ETH en quichua santiagueño, atendiendo a su forma, distribución y función en el discurso. El resto del artículo, por tanto, se organiza como sigue. En §2, se brinda un breve perfil tipológico de la lengua, en especial de aquellos aspectos de relevancia para la discusión posterior. En $\S 3$, se desarrolla el marco teórico del trabajo. En §4, se mencionan algunas consideraciones metodológicas. En $\$ 5$ se describen y analizan las diversas construcciones de ETH en la lengua. En $§ 6$, por último, se presentan las conclusiones.

\section{EL QUICHUA SANTIAGUEÑO}

El quichua santiagueño es una lengua de la familia quechua hablada en Argentina, sobre todo en la provincia de Santiago del Estero y, como resultado de la migración interna, en otras grandes ciudades del país. La lengua se inscribe dentro de la rama 
quechua IIC o quechua chinchay meridional (Cerrón-Palomino, 2003) ${ }^{1}$. Se estiman unos 160.000 hablantes en la provincia de Santiago del Estero y unos 150.000 en Buenos Aires y otras zonas (Albarracín, 2011; Alderetes, 2001).

Como el resto de las lenguas de la familia, el quichua santiagueño se caracteriza por una morfología sufijante y aglutinante. Su alineamiento morfosintáctico es nominativo-acusativo. Existen dos grandes clases de palabras: verbo y nombre (que comprende también adjetivos y adverbios). Respecto del orden de palabras, el quichua santiagueño exhibe el orden SOV típico de las lenguas quechua (Cerrón-Palomino, 2003), pero con un margen de flexibilidad considerable. Esto último se replica en el orden cláusula dependiente-cláusula principal.

La principal estrategia para la codificación de eventos dependientes es la nominalización, manifestada en completivas, relativas y adverbiales. Algunos sufijos nominalizadores que codifican estas últimas integran a la vez un sistema de conmutación de la referencia (switch-reference; CR de aquí en más), señalando relaciones temporales, de concesión, condición, razón y circunstancia (para los sistemas de CR en general, véase Haiman y Munro, 1983 y Stirling, 1993; para el quichua santiagueño, Juanatey, 2016 y 2020). Los marcadores de CR alternan para evitar la ambigüedad entre referentes copresentes, al señalar morfosintácticamente (dis)continuidad referencial entre las cláusulas relacionadas. Además del seguimiento referencial, este sistema puede indicar (dis)continuidad temporal entre los eventos. La cláusula marcada (dependiente) es la que lleva el sufijo de $\mathrm{CR}$, mientras que la cláusula principal (finita) es la de referencia o control. Este sistema es indexado en quichua santiagueño mediante los sufijos nominalizadores -spa CONT (4) y -pti DISC (5) ${ }^{2}$.

(4) Continuidad de referente

$\begin{array}{llll}\text { [yacha-spa } a_{\mathrm{i}} & \text { kutis-ni-yki] } & \text { ancha-mi } & k u s i-k u-n i_{\mathrm{i}} \\ \text { saber-CONT } & \text { permanencia-EUF-POS.2SG } & \text { muy-VAL } & \text { alegrar-RFL-1SG }\end{array}$

(5)

"Saber de su presencia, me alegra mucho".

Discontinuidad de referente

$\begin{array}{llll}\text { [volya-pti-yki] } & \text { tiya- } n_{\mathrm{j}} & \text { cuida-na-paq } & \text { uywa-s-ta } \\ \text { volver-DISC-POS.2SG } & \text { COP.LOC-3SG } & \text { cuidar-NMLZ.POST-BEN } & \text { animal-PL-AC }\end{array}$

"Cuando vuelvas, habrá animales para cuidar".

${ }^{1}$ El quechua IIC se extiende por Perú (departamentos de Huancavelica, Ayacucho, Apurímac, Arequipa, Cuzco, Puno y Moquegua), Bolivia, Argentina y la provincia chilena de Antofagasta (Cerrón-Palomino. 2003, p. 242)

2 Considerando los aportes de Stirling (1993) y van Gijn y Hammond (2016) acerca de la complejidad funcional de los sistemas de CR (ver §3), emplearemos las nociones de "continuidad" (CONT) y "discontinuidad" (DISC) en lugar de las más tradicionales "mismo sujeto" y "distinto sujeto" (Haiman y Munro, 1983).

314 | AlPHA № 53 (Diciembre 2021) PÁGS. 311-329. ISSN 07 16-4254 
A su vez, la lengua cuenta con una forma verbal reducida, señalada mediante el sufijo $-s$, llamada aquí converbo (Juanatey, 2016 y 2020) . $^{3}$ Al igual que el sufijo -spa del sistema de CR, $-s$ señala continuidad referencial. Se diferencia, sin embargo, por establecer relaciones adverbiales más estrechas desde el punto de vista sintácticosemántico con el verbo principal: en general, circunstancia (inmediata), manera, fase o modificación aspectual, entre otras. En (6) se ilustra la relación de manera de movimiento.

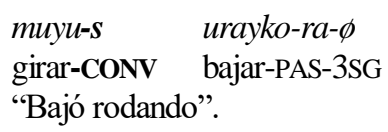

Como se verá, las cláusulas de CR y las formas converbales son los tipos de construcciones que suelen aparecer como cabezas de ETH.

\section{MARCO TEÓRICO}

De Vries define el ETH como "un modo de conectar cadenas clausales en el que la última cláusula de la cadena es parcial o por completo repetida en la primera cláusula de la cadena siguiente" (2005, p. 363; nuestra traducción). En esta definición se ponen de manifiesto dos aspectos de las construcciones de ETH. Por un lado, su perfil formal en tanto repetición de la cláusula anterior. Por el otro, su carácter funcional como recurso de conexión entre cláusulas.

Respecto de la forma de las construcciones de ETH, en $§ 1$ se mostró que estas pueden exhibir una variación considerable. De hecho, es posible distinguir dos grandes tipos. En primer lugar, se reconocen las construcciones que involucran la repetición exacta del predicado anterior, como se ve en (2), denominadas verbatim (de Vries, 2005) o de recapitulación (Aikhenvald s.f); estas son las construcciones de ETH canónicas. En segundo lugar, se identifican construcciones no verbatim, es decir, que retoman el contenido proposicional de la cláusula previa pero sin repetir el mismo lexema. Esto puede materializarse con el uso de un predicado genérico, del tipo 'hacer' o 'estar', a veces acompañado por un elemento deíctico como un demostrativo. El ejemplo (3) del quichua santiagueño con la forma chayna ris 'así yendo' es un caso de esto. Estas construcciones de ETH son llamadas genéricas (de Vries, 2005) o de resumen (Aikhenvald, s.f).

En relación con esto, van Gijn (2014) propone un continuum de solapamiento (overlap) semántico entre los eventos denotados por las cláusulas de la cola y la cabeza,

\footnotetext{
3 Según Cerrón-Palomino (2003, p. 196), otras lenguas de la familia como el argentino, el colombiano y los quechua orientales del Ecuador, también presentan un sufijo - $s$. El autor no señala si estas formas cuentan con alguna especificación funcional, sino que se las incluye como formas apocopadas de -spa o sufijos similares.
} 
ilustrado en la Figura 1. En uno de los extremos se ubican las construcciones de repetición verbatim, que exhiben un solapamiento máximo con el predicado anterior. A continuación se emplazan las construcciones con verbos genéricos, con un grado menor de solapamiento. Luego se reconocen construcciones con formas parafrásticas, donde se recurre a un predicado más específico que reformula al anterior. Luego, en una posición marginal, se localizan las construcciones con predicados que denotan un evento fácilmente asociado o deducible del previo (como puede ser su consecuencia o un estado resultante inmediato). Más allá de estas últimas, se diluye el solapamiento semántico entre los eventos.

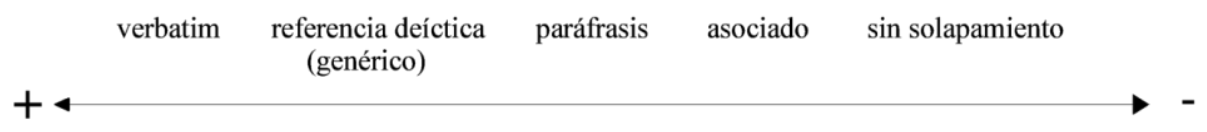

Figura 1. Construcciones ETH según grado de solapamiento con el evento previo (tomado de Van Gijn, 2014, p. 297)

Es plausible que las particularidades morfosintácticas de las distintas lenguas permitan reconocer subtipos adicionales dentro de esta tipología. Como se verá en la sección §5, la distinción entre cláusulas de CR y converbos del quichua santiagueño da lugar a distintos subtipos dentro de este continuum.

En cuanto a la función de estas construcciones, la definición de de Vries (2005) resalta su rol para la conexión entre cadenas clausales, estas últimas entendidas como las unidades mínimas de discurso multiproposicional coherente (Givón, 2001, p. 355). La coherencia temática remite a la continuidad o recurrencia de varios elementos codificados por el léxico o la gramática (Givón, op. cit., pp. 328-329): a) referentes, b) locación, c) temporalidad, aspectualidad, modalidad, e) perspectiva, y f) acción/eventos. La continuidad de uno de estos elementos - por ejemplo, los referentes- puede interrumpirse en algún punto del discurso sin que se suspenda por completo la coherencia temática; esta es, en definitiva, una cuestión de grado. Nótese, asimismo, que la continuidad de "acción/eventos" puede interpretarse como el solapamiento semántico del continuum de van Gijn (2014) de la Figura 1.

En su análisis de las construcciones de ETH en las lenguas papuanas, De Vries (2005) concluye que estas tienen cuatro funciones principales: a) el facilitamiento del procesamiento textual, b) la coherencia referencial, c) la continuidad temática y d) la discontinuidad temática ${ }^{4}$. Estas funciones están en sintonía con la visión en los estudios

\footnotetext{
${ }^{4}$ La aparente contradicción de las últimas dos funciones se debe a que algunas lenguas manifiestan más de un tipo de construcción de ETH. Si bien su función elemental es la de favorecer la continuidad temática, algunas de ellas provocan el efecto contrario de señalar un quiebre en la continuidad como, por ejemplo, al comienzo de un nuevo episodio (de Vries, 2005, p. 8).
} 
tipológicos de que las cláusulas adverbiales en posición inicial colaboran en la organización y coherencia discursiva (Chafe, 1984; Thompson, Longacre y Hwang, 1985; Thompson, 1987; Matthiessen y Thompson, 1988). Se ha observado que este tipo de cláusulas "tienden a tener conexiones más globales, difusas y pragmáticas con su contexto discursivo, y están por ende menos integradas a la estructura semántica de la cláusula principal" (Givón, 2001, p. 343; nuestra traducción). En este respecto, resulta de interés observar si los distintos tipos de construcciones de ETH varían en su grado de integración eventiva con la cláusula principal (Van Valin, 2005) y cuál puede ser el efecto más general de esto para la coherencia temática.

\section{CONSIDERACIONES METODOLÓGICAS}

Para el estudio de las construcciones de ETH en quichua santiagueño se analizó un corpus de 20 narrativas de fuentes primarias y secundarias. Los datos primarios constituyen narraciones elicitadas en los años 2013-2018 a hablantes de entre 30 y 60 años en la provincia de Santiago del Estero y diferentes barrios del oeste y sur del Gran Buenos Aires. Los datos de fuentes secundarias, por su parte, fueron recuperados de textos de relativa antigüedad, como Bravo (1965), la antología de textos históricos y contemporáneos Tebes y Karlovich (2010), textos literarios (como Tevez en Albarracín 2016), y producciones de hablantes jóvenes (como Guillín et al. 2012). En todos los casos los textos corresponden a hablantes bilinguies quichua-español.

\section{CONSTRUCCIONES DE ETHEN QUICHUA SANTIAGUEÑO}

Siguiendo el continuum de la Figura 1, a continuación se distinguen cláusulas que funcionan como cabeza de ETH según el solapamiento con el evento anterior. Así, en §5.1 se abordan las construcciones verbatim y en $\$ 5.2$ las no verbatim. Dentro de cada una se reconocen subtipos formales y se los caracteriza funcionalmente.

\subsection{CONSTRUCCIONES DE ETH VERBATIM}

Las construcciones de ETH verbatim en el quichua santiagueño exhiben tres manifestaciones formales distintas: la forma con el sufijo converbal $-s$, la forma con el sufijo de continuidad -spa y la forma con el sufijo de discontinuidad -pti. Recuérdese que tanto el converbo como la forma con -spa anticipan continuidad (en general correferencia de sujetos), en contraposición con el sufijo -pti.

Tómese el siguiente ejemplo, extraído de un texto que narra la resucitación de un hombre. En (7) se relata su regreso del cielo a la tierra.

$$
\begin{aligned}
& \text { a. } \quad y \quad r i-p t e-n-q a \\
& \text { y ir-DS-POS.3SG-TOP } \\
& \text { karu-lla-manta }
\end{aligned}
$$




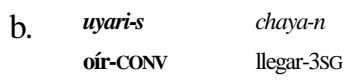

"Escuchándolos llegó".

(Bravo, 1965, p. 137)

En (7.b) se repite el verbo uyari- "oír" de la cláusula finita de (7.a) como una forma converbal con el sufijo $-s$. Esta repetición resalta que durante el viaje de vuelta el personaje no deja de escuchar los llantos de los dolientes en su propio velorio. Centralmente, además, el converbo uyaris exhibe un alto grado de integración semántica con el predicado principal, como modificador adverbial de manera de chayan "llegó".

Otro ejemplo es el siguiente, también parte de una narración de resucitados (Bravo, 1965).

a.

wañu-chi-pti-n

wañu-s

$\underline{r i-s a}$

ir-NMLZ.ANT

$\underline{k a-r a-\phi}$

"Cuando lo había matado, muerto él había ido"

b.

$\begin{array}{llccc}r i-s & q a a-s a & k a-r a-\phi & c h a & \text { riña-s-t } \\ \text { ir-CONV } & \text { ver-NMLZ.ANT } & \text { COP-PAS-3SG } & \text { DEM.MED } & \text { riña-PL-AC } \\ & & \text { entero } & \text { canilla-nkuna } & \text { puka-lla } \\ \text { S.3PL-AC } & & \text { entero } & \text { canilla-POS.3PL } & \text { rojo-EXCL }\end{array}$

$\begin{array}{llll}\text { puri-sqa-nkuna-ta } & \text { entero } & \text { canilla-nkuna } & \text { puka-lla } \\ \text { andar-NMLZ.ANT-POS.3PL-AC } & \text { entero } & \text { canilla-POS.3PL } & \text { rojo-EXCL }\end{array}$

"y yendo había visto a esos que hacen riñas, que andaban en penitencia, toda la pierna roja de sangre nomás".

(Bravo, 1965, p. 135).

En (8) se narra el deambular del alma del protagonista por diferentes escenarios. El converbo del verbo "ir" de (8.b) señala una continuidad en el desplazamiento iniciado en (8.a). Como en el ejemplo anterior, el converbo exhibe un alto grado de integración semántica con el evento qaasa kara "había visto" de la principal, estableciendo una relación de simultaneidad.

En otras construcciones, el predicado que repite el verbo anterior puede llevar el sufijo de continuidad -spa o el de discontinuidad -pti. Es de notar que el primero es menos frecuente en nuestro corpus que el segundo. Consíderese el siguiente ejemplo:

\begin{tabular}{|c|c|c|c|c|c|c|}
\hline \multirow[t]{2}{*}{ a. } & api-s & vejiga-ta & \multicolumn{2}{|l|}{$\underline{\text { unta-chi-sa }}$} & \multicolumn{2}{|l|}{$\underline{k a-r a-\phi}$} \\
\hline & tener-CONV & vejiga-AC & $\underline{\text { lenar-CAUS-1 }}$ & & COP-PAS-3SG & \\
\hline b. & $\begin{array}{l}\text { "Agarrándolas } \\
\text { unta-chi-spá } \\
\text { llenar-CAus-CoNT.TOP }\end{array}$ & $\begin{array}{l}\text { había llenado } \\
\text { chaguar-situ- } t \\
\text { chaguar-DIM-AC }\end{array}$ & $\begin{array}{l}\text { vejiga". } \\
\text { kuyu-s } \\
\text { torcer-Conv }\end{array}$ & $\begin{array}{l}\text { vejiga-t } \\
\text { vejiga-AC }\end{array}$ & $\begin{array}{l}\text { simi-n-ta } \\
\text { boca-Pos.3SG-AC }\end{array}$ & $\begin{array}{l}\text { wata-pu- } \phi-s \\
\text { atar-APL-OB.3-CONV }\end{array}$ \\
\hline & $\begin{array}{l}\text { law } \\
\text { lado }\end{array}$ & $\begin{array}{l}\text { punta-n-an } \\
\text { punta-Pos.3sG-Com }\end{array}$ & $\begin{array}{l}\text { apa-s } \\
\text { llevar-CONV }\end{array}$ & $\begin{array}{l}\text { chupa-n-pi } \\
\text { cola-POS.3sG-LOC }\end{array}$ & $\begin{array}{l}\text { wata-pu- } \phi \text {-s } a \\
\text { atar-APL-OB.3-NMLZZANT }\end{array}$ & $\begin{array}{l}\frac{k a-r a-\phi}{\text { COP-PAS-3SG }} \\
\underline{\text { nas }}\end{array}$ \\
\hline
\end{tabular}


Construcciones de enlace tail-head en quichua santiagueño

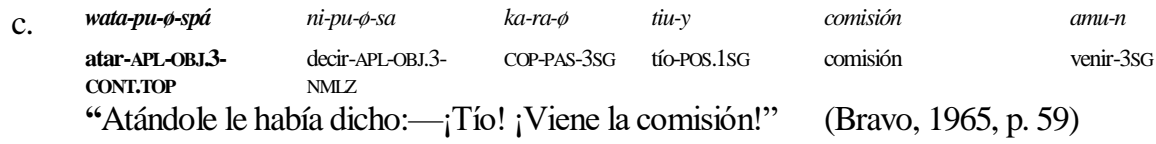

El fragmento anterior es parte de una fábula referente a una disputa por comida entre el zorro y el tigre. En este punto se describe el proceso de ejecución del artilugio distractor que crea el zorro. En (9.a) este último llena una vejiga de vaca con moscas, untachisa kara "la había llenado". En (9.b) este predicado es retomado por untachispá "llenándola", que tiene la marca de continuidad -spa. Lo mismo sucede con el predicado watapusa kara "le había atado" y su repetición watapuspá "atándole" en (9.b).

Obsérvese otro ejemplo tomado de un texto donde una mujer enumera sus tareas domésticas:

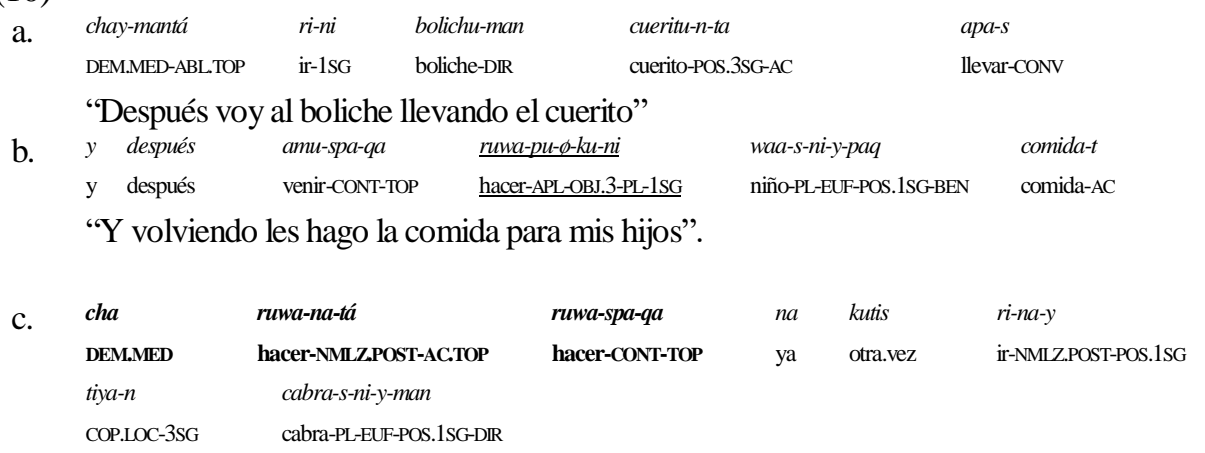

"Haciendo ese quehacer ya tengo que ir otra vez a mis cabras".

(Bravo, 1965, p. 65)

En (10.b) la mujer cuenta que al llegar del almacén, hace la comida para sus hijos, y en (10.c) retoma esto último con la forma ruwaspaqa "haciendo". Por último menciona la siguiente tarea de su itinerario, ir a cuidar a sus cabras.

Tanto en (9) como en (10) se observa que las cláusulas verbatim con -spa se emplazan en secuencias no solo de alta continuidad referencial, sino temática (Givón, 2001; véase §3). En los ejemplos se concatenan eventos de un mismo participante que constituyen una unidad episódica, como lo son las etapas de un proceso (9) o itinerario (10). Creemos que la acción conjunta de la repetición verbatim y el sufijo de continuidad en la cláusula cabeza favorecen esta función.

Por último, la tercera variante de ETH verbatim son las formas con el sufijo de discontinuidad -pti, como se ve en (11). 
(11)

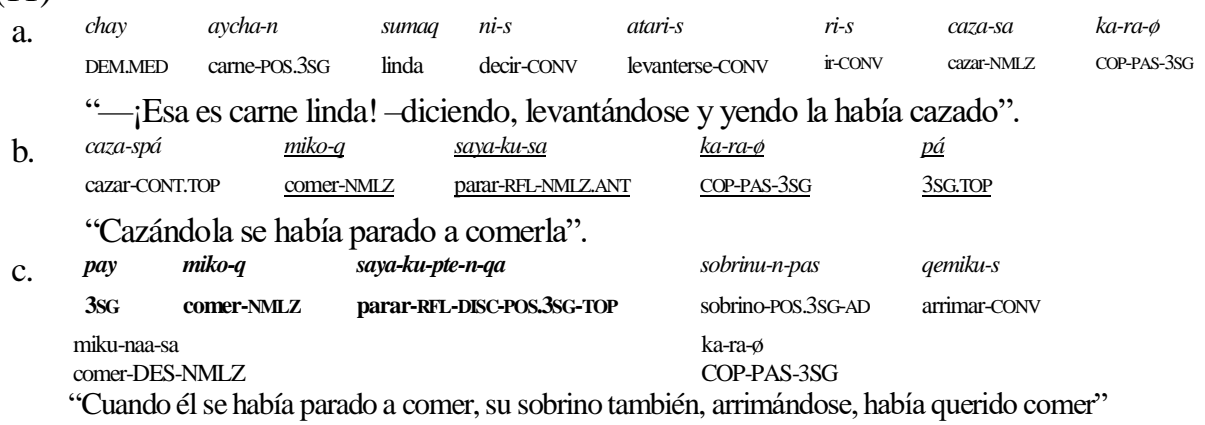

(Bravo, 1965, p. 59)

Este pasaje, tomado del mismo relato de (9), narra cómo el tigre caza una vaca y se dispone a comerla en (11.b). Esto último se retoma en (11.c), pero esta vez con el sufijo -pti, que como marca de discontinuidad desplaza la atención al otro participante, el sobrino (el zorro), que se arrima.

Considérense, además, los dos siguientes ejemplos, provenientes del texto sobre el resucitado de (7). En (12) se inicia el relato.
a. พап̃u-s
$\frac{\text { lloqse-ra- } \phi}{\text { salir-PAS-3SG }}$
wasi-n-manta
morir-CONV
ma) salió de su casa".
b. wasi-n-manta
casa-POS.3SG-ABL
lloqsi-pte-n-qa
salir-DISC-POS.3SG-TOP
ishkay
dos
palomitas
ina
ñawpa-pu- $\phi-s$
re-ra-nku

"Cuando salió de su casa dos palomitas le fueron detrás".

(Bravo, 1965, p. 135)

En (12.b) se retoma la partida del alma con wasinmanta lloqsiptenqa "cuando salió de su casa", con el sufijo de discontinuidad -pti. Esta marca permite la introducción de dos participantes nuevos: las dos palomitas que lo acompañan en el ascenso al cielo.

Luego, en su viaje de retorno a la tierra, el personaje se percata de que su burra está dando a luz lejos de la casa. Al resucitar informa esto a su familia.

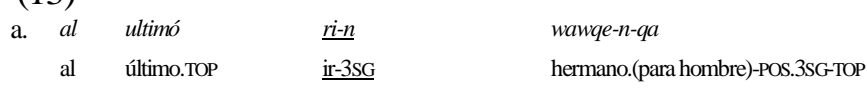

"Al final va su hermano"

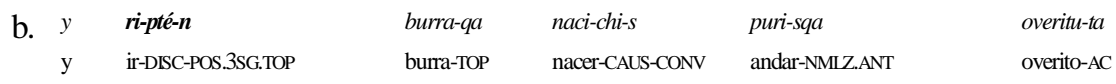

"Y cuando va la burra había andado pariendo un overito" ～(Bravo, 1965, p. 135)

320 | AlPHA № 53 (DicIEMBre 2021) PÁGS. 311-329. ISSN 07 16-4254 
En (13.a) se narra el momento en el que el hermano del resucitado va al monte a buscar a la burra. En (13.b) se retoma el verbo rin 'va' con riptén 'cuando va'. Así, el ETH con sufijo de discontinuidad habilita el cambio de atención desde el hermano a la burra.

En la literatura tipológica (Stirling, 1993; van Gijn, 2016), las marcas de discontinuidad de los sistemas de CR han sido descriptas como funcionalmente complejas, ya que no indican solo discontinuidad referencial. Para las construcciones de ETH, Stirling (1993) argumenta que esta discontinuidad puede desempeñar funciones tales como el señalamiento de fronteras discursivas -como ocurre en (11), donde la cláusula cabeza marca una transición en la narración hacia el conflicto central del relato-; la introducción de nuevos participantes -como en (12), donde se presentan las palomitas que acompañan al alma del muerto-; o anticipar desenlaces excepcionales -como en (13), donde se confirma el vaticinio del resucitado-.

En síntesis, las construcciones de ETH de repetición verbatim establecen por definición un alto grado de continuidad temática con el discurso previo, como resultado del solapamiento entre los predicados de las cláusulas cola y cabeza. Se vio, asimismo, que en quichua santiagueño se verifican tres subtipos dentro de este grupo: a) converbales, b) con sufijo -spa, y c) con sufijo -pti. Estos se distinguen por su grado de integración sintáctico-semántica con el predicado principal. Las formas converbales suponen relaciones entre eventos más estrechas, notablemente de manera (7) o simultaneidad (8). Las formas con sufijo -spa, por su parte, implican un considerable grado de continuidad en términos de correferencia o integración eventiva con la cláusula matriz, indicando, por ejemplo, secuencialidad en procedimientos (9) o itinerarios (10). Las formas con el sufijo -pti, por último, implican no solo menos integración con el evento principal, sino que señalan puntos de discontinuidad en términos discursivos más amplios, como se vio en los ejemplos (11-13).

\subsection{CONSTRUCCIONES DE ETH NO VERBATIM}

El análisis del corpus mostró que las construcciones de ETH no verbatim en quichua santiagueño exhiben distintas variantes del continuum presentado en la Figura 1 en §3. De manera más específica, se constataron formas genéricas y formas parafrásticas.

Es muy frecuente que además de usarse para repeticiones verbatim, el converbo del verbo "ir" ris se utilice como enlace general de otros verbos de movimiento, es decir, en construcciones de ETH genéricas (de Vries, 2005) ${ }^{5}$. Considérese el ejemplo (3), renumerado aquí como (14).

\footnotetext{
5 A la vez, se identificó en el corpus con mucha menos frecuencia la forma converbal tiyas de la cópula locativa, que también parece funcionar como forma genérica (véase Juanatey 2020 para profundizar acerca de esta estrategia).
} 
(14)
a. lloqsi-sa
$\underline{\text { ka-ra- } \phi}$
salir-NMLZ.ANT
COP-PAS-3SG
"Salió caminando despacito".
marcha-s
allim:itan-ta
marchar-CONV
despacio:DIM-ADVR
b.
$\begin{array}{lll} & r i-s & \text { tari-sa } \\ \text { DEM.MED-COMP } & \text { ir-CONV } & \text { encontrar-NMIZ.ANT }\end{array}$
$\begin{array}{lll}k a-r a-\phi & s u k & \text { huell-ita-ta } \\ \text { COP-PAS-3SG } & \text { una } & \text { huella-DIM-AC }\end{array}$

"Así yendo, encontró una huellita"

(Tevez en Albarracín 2016)

En (14.b) el converbo ris retoma el predicado de movimiento anterior lloqsisa kara "salió" de (14.a), pero sin establecer una repetición exacta. Nótese que ris va acompañado por el demostrativo chayna "así/como eso", un rasgo muy común de las construcciones de ETH genéricas (de Vries, 2005), ya que orienta la atención del interlocutor de manera endofórica hacia lo mencionado antes.

En otros casos, el converbo ris puede recapitular varios eventos de movimiento, mostrando un alcance mucho más amplio que en el ejemplo anterior. Esto se ve en (15), que es parte de un relato donde el narrador cuenta su primer viaje en un automóvil.

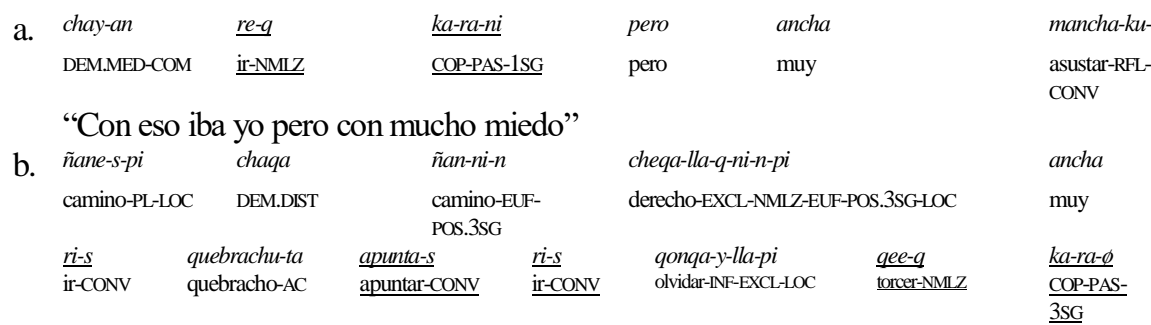

"En los caminos, en las rectas del camino, yendo a gran velocidad, apuntando a un quebracho, en un descuido, solía torcer"

$\begin{array}{lllllll}\text { c. y } & \text { qee-chi-pti-n } & \text { respira- } q & k a-r a-n i & \text { chayraq } & \text { chaqa } & \text { hombre } \\ \text { y } & \text { torcer-CAUS-DISC-POS.3SG } & \text { respirar-NMLZ } & \text { COP-PAS-1SG } & \text { todavía } & \text { DEM.MED } & \text { hombre } \\ & \text { erra-pu- } \phi \text {-pti-n } & \text { quebrachu-tá } & & & \\ \text { errar-APL-OBJ.3-DISC-POS.3SG } & \text { quebracho-AC.TOP } & & & \end{array}$

"Y cuando torcía recién solía respirar yo, o cuando aquel hombre le erraba al quebracho"

d. cha-yna urayko-ra-yku chaqa administracion-pi DEM.MED-COMP ir-CONV bajar-PAS-1PLEXCL DEM.DIST administración-LOC

"Yendo así nos bajamos en la administración" （Bravo, 1965, p. 203)

Aquí la construcción chayna ris en (15.d) adopta la función de resumen señalada para las construcciones genéricas por Aikhenvald (s.f.) y van Gijn (2014). En este sentido, marca el límite final de la secuencia del recorrido, anticipando un cambio de locación: la llegada a la administración en (15.d), donde concluye el viaje. 
A menudo, estas construcciones con ris pueden recapitular eventos que no son de movimiento. Considérese el siguiente ejemplo.
a. unay-qa música ka-q ka-ra- $\phi$
hace.tiempo-TOP música COP-NMIZ COP-PAS-3SG
'Hace tiempo la música solía ser":
b. arpa-ta

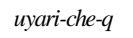
ka-ra- $\phi$
waño-q
arpa-AC
morir-NMLZ
Gervasio.Contreras
Gervasio.Contreras
oír-CAUS-NMIZ
COP-PAS-3SG
"El arpa solía tocarla el finado Gervasio Contreras"

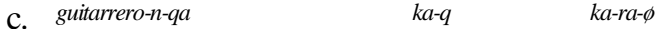
guitarrero-POS.3SG-TOP COP-NMEZ COP-PAS-3SG Santiago.Coria
"El guitarrero era Santiago Coria".
d. bombisto-n-qa ka-q ka-ra- $\phi \quad$ Eusebio.Jiménez
bombisto-POS.3SG-TOP COP-NMLZ COP-PAS-3SG Eusebio.Jiménez
"Su bombisto era Eusebio Jiménez"
e. $y$ noqa ri-s violin-ta aprende-ra-ni
y $1 \mathrm{SG} \quad$ ir-CONV violín-AC apreder-PAS-1SG
"Y yo (yendo) aprendí el violín".
(Bravo, 1965, p. 226)

En (16.e) el converbo ris resume el contexto en el que el narrador aprendió a tocar el violín (16.a-d). Aquí se ve que las formas genéricas pueden tener un alcance discursivo mayor que el de las formas verbatim, de ahí su nombre alternativo de construcciones de "recapitulación" (Aikhenvald, s.f.).

Por último, también es posible encontrar formas que, sin replicar el verbo anterior, tampoco constituyen casos de verbos genéricos. Se destacan, no obstante, por retomar la oración previa mediante una paráfrasis o reformulación del evento. El siguiente es un extracto de una narración de la aparición del espectro de una mujer.
a. abuelo-y
abuelo-POS.1SG
chaymant
volya-ku-sa
volver-RFL-NMIZ.ANT
"Mi abuelo entonces había vuelto por otro lado"
$k a-r a-\phi$ suk law

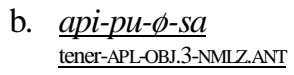
$\underline{\underline{\text { COP-PAS- }-3 \mathrm{SG}}}$

\section{$\underline{\text { chiri.chiri }}$}
"Le había dado un escalofrío"
c.
asustar-RFL-DIS-POS.3SG
kutis
warmi
ñawqe-man
chura-ku-pu- $\phi$-sa
$k a-r a-\phi$
"Y cuando tenía miedo otra vez la mujer se le puso adelante" (Guillín et al., 2012, p. 34)

Así, en (17.c) manchakuptin "cuando tenía miedo" es una reformulación de la oración anterior apipusa kara chiri chiri "le dio un escalofrío" en (17.b). La marca de discontinuidad -pti, además de indicar la reaparición del personaje de la mujer, anticipa un cambio de episodio. Estas instancias de reformulación aparecen solamente con este sufijo en el corpus analizado. 
En suma, en quichua santiagueño se identificaron en particular dos posibilidades formales para construcciones de ETH no verbatim: a) el converbo del verbo ri- "ir", ris (en algunos casos precedido por un demostrativo), y b) una forma con el sufijo -pti de discontinuidad. Ambas estrategias se diferencian por el grado de solapamiento que sostienen con el predicado de la cláusula cola de la oración previa. El converbo ris puede actuar como un verbo genérico, ya sea retomando eventos de movimiento o no. Las cláusulas con marca de discontinuidad -pti, en cambio, pueden ser paráfrasis o reformulaciones de las anteriores. De esta manera, a nivel discursivo establecen diferentes instancias de continuidad temática en relación con el contexto precedente.

Van Gijn (2014) advierte que las cláusulas cabeza de las construcciones de ETH presentan la información anterior como indicadores de transiciones discursivas. Así, pueden señalar un cierre de secuencia y cambio a una locación de importancia para el relato -como en (15), donde el protagonista desciende finalmente del automóvil-, la síntesis de una situación -como en (16), donde se recuerda a los músicos de la época-, o solo un cambio de atención hacia un participante importante -como en (17), donde reaparece el espectro de la mujer-.

\section{DISCUSIÓN Y CONCLUSIONES}

El objetivo de este trabajo fue ofrecer una caracterización preliminar de las construcciones de ETH en quichua santiagueño. En primer término, se constataron dos tipos de construcciones: a) las verbatim, que implican la repetición exacta del verbo anterior, y b) las no verbatim, que no repiten el verbo anterior, sino que lo retoman de distintas maneras. En las primeras se reconocieron tres formas para las cláusulas cabeza: a) converbales con el sufijo $-s$, b) con el sufijo de continuidad -spa, y c) con el sufijo de discontinuidad -pti. En las segundas, se identificaron construcciones con la forma genérica ris (el converbo del verbo "ir") y parafrásticas o de reformulación con -pti.

Propusimos dos parámetros para caracterizar las variantes de estas construcciones. El primero involucra el grado de solapamiento semántico que la cláusula cabeza manifiesta con la anterior (van Gijn, 2014), describiendo un continuum. Este parámetro contempla la función de estas construcciones al nivel del discurso. Asimismo, argumentamos que un parámetro de clasificación adicional es el grado de integración eventiva que la cláusula cabeza posee en tanto adverbial prepuesta con el predicado principal. Nótese que este criterio se complementa con el anterior puesto que toma en cuenta la función de las construcciones al nivel de la sintaxis. Ambos parámetros se ilustran en la Figura 2. 


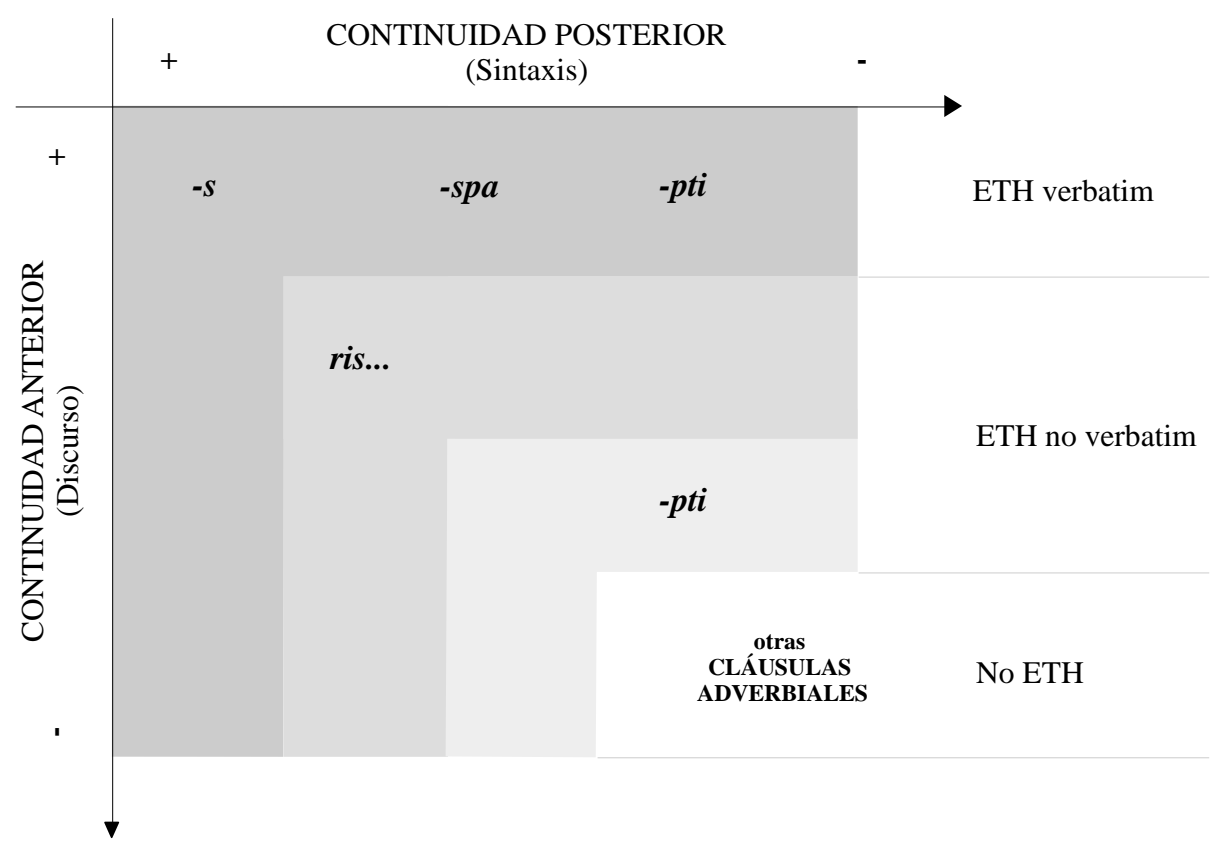

Figura 2. Rol de las construcciones ETH en quichua santiagueño en la coherencia temática

El eje vertical muestra la distribución de las formas según el primer parámetro, de ahí que las construcciones verbatim, que replican el contenido exacto, ocupen la posición superior; estas se ven seguidas por las no verbatim -las construcciones genéricas, y luego las parafrásticas-. El eje horizontal, por su parte, grafica el segundo parámetro. Las construcciones con converbo $-s$ se localizan en el extremo izquierdo debido a que indican relaciones como manera o simultaneidad, que son las que exhiben mayor integración eventiva. Las formas con el sufijo de continuidad -spa, por su parte, suelen implicar no solo identidad referencial sino también relaciones de simultaneidad o secuencialidad. Porúltimo, en el extremo derecho, las construcciones con el sufijo de discontinuidad -pti muestran falta de correferencia $\mathrm{y}$, también, pueden señalar interrupciones en la continuidad de otros elementos temáticos, como cambios en la temporalidad, locación, y otras.

La interacción de los dos parámetros permite reconocer diferentes funciones discursivas de cada variante de acuerdo con su rol en la coherencia temática. Como la define Givón (2001), esta última es producto de la (dis)continuidad de elementos diversos, como los referentes, la locación y las acciones/eventos, entre otros (véase §3). Así, entre las construcciones verbatim, la forma converbal favorece un alto grado de coherencia, como puede ocurrir en el relato de un recorrido, como se vio en el ejemplo (8), o al señalar la manera de un desplazamiento (7), porque garantiza la identidad 
referencial y una estrecha integración eventiva. La forma con sufijo de continuidad -spa también se emplaza en puntos de alta coherencia, indicando identidad referencial y una considerable integración eventiva, como se da en la descripción de los pasos de un procedimiento (9) o un itinerario (10). La forma con sufijo de discontinuidad -pti, por su parte, también propicia la extensión de la coherencia temática con el contexto previo, pero anticipa por definición un cambio de referente, que puede desplazar la atención a otro participante $(11,12)$ o marcar un límite discursivo (13).

Las construcciones de ETH no verbatim, en cambio, favorecen una discontinuidad más acentuada en la coherencia temática. De este modo, la forma genérica ris puede señalar un cambio de locación $(14,15)$ o recapitular una situación previa que sirve de contexto para los sucesos subsiguientes (16). Debe notarse que el alcance de la unidad discursiva que retoma es significativamente mayor. La forma no verbatim parafrástica, por último, implica una interrupción más marcada en la coherencia temática, máxime porque solo se manifiestan con el sufijo de discontinuidad -pti (17).

La descripción de las construcciones de ETH del quichua santiagueño inspira algunas preguntas de investigación adicionales. Resulta pertinente ahondar en el inventario de las estrategias de la lengua que configuran el seguimiento de la coherencia temática en el discurso multiproposicional y evaluar qué lugar ocupan construcciones de ETH en este respecto. Es preciso, por tanto, examinar más de cerca el rol de otras cláusulas adverbiales prepuestas, por ejemplo, y el funcionamiento de dispositivos de seguimiento referencial aquí no contemplados. Otro interrogante que se suscita es la distribución de las construcciones de ETH en distintas variedades, registros y géneros de la lengua. Como se vio aquí, estas son muy frecuentes en narrativas. Hemos notado, no obstante, que podrían encontrarse en proceso de remisión en la oralidad de los narradores más jóvenes. Es oportuno preguntarse, por ejemplo, si esto se sostiene en todos los géneros y registros. Las futuras investigaciones pueden contribuir a echar más luz acerca de aspectos como estos.

\begin{abstract}
ABREVIATURAS
1 primera persona; 2 segunda persona; 3 tercera persona; $\mathrm{ABL}$ ablativo; $\mathrm{AC}$ acusativo; AD aditivo; ADVR adverbializador; ANT anterior; APL aplicativo; BEN benefactivo; CAUS causativo; COM comitativo; COMP comparativo; CONT continuativo; CONV converbo; COP cópula; DEM demostrativo; DIM diminutivo; DIR direccional; DISC discontinuidad; DIST distal; DS diferente sujeto, EUF eufónico; EXCL exclusivo; GEN genitivo; INF infinitivo; LOC locativo; MED medial; NF no futuro; NMLZ nominalizador; PAS pasado; PL
\end{abstract}


plural; POS posesivo; POST posterior; RFL reflexivo; SG singular; TOP topicalizador; TR sonido transicional; VAL validativo.

\section{OBRAS CITADAS}

Adelaar, Willem F. H; Pieter Muysken (2004). The Languages of the Andes. Cambridge University Press.

Aikhenvald, Alexandra (s.f). Bridging linkage in Tariana, an Arawak language from north-west Amazonia. En https://www.academia.edu.

Albarracín, Lelia I. (2011). La Quichua: gramática, ejercicios y diccionario QuichuaCastellano. Vol. 2. Dunken.

Alderetes, Jorge R. (2001). El Quechua de Santiago del Estero, gramática y vocabulario. Universidad Nacional de Tucumán.

Bravo, Domingo A. (1965). Estado actual del quichua santiagueño. Universidad Nacional de Tucumán, Facultad de Filosofía y Letras.

Cerrón-Palomino, Rodolfo (2003). Lingüística quechua. Centro de Estudios Rurales Andinos "Bartolomé de las Casas".

Chafe, Wallace (1984). How people use adverbial clauses. Annual Meeting of the Berkeley Linguistics Society 10 (0), 437-449.

De Vries, Lourens (2005). Towards a typology of tail-head linkage in Papuan languages. Studies in Language. 29 (2), 363-384.

Dixon, Robert M. W (2009). The semantics of clause linking in typological perspective. En: A. Aikhenvald; R. M. W. Dixon (eds.) The Semantics of Clause Linking: A Cross-Linguistic Typology. Oxford, UK: Oxford University Press, 1-55.

Givón, Talmy (2001). Syntax: An Introduction. Vol. 2. John Benjamins.

Guillaume, Antoine (2011). Subordinate clauses, switch-reference, and tail-head linkage in Cavineña narratives. En: R. van Gijn, K. Haude; P. Muysken (eds.) Subordination in native South-American languages. (pp. 109-139). John Benjamins.

Guillín, César; Orlando López; Azucena Torrez; Mariela Pérez; Richar Guillín; Elías Barraza (2012). Wawqes Pukllas. Libro juvenil quichua. En el aura del sauce.

Haiman, John; Pamela Munro (eds.) (1983). Switch-Reference and universal grammar: Proceedings of a symposium on switch reference and universal grammar, Winnipeg, May 1981. John Benjamins.

Haiman, John; Thompson, Sandra A. (eds.) (1988). Clause Combining In Grammar And Discourse. John Benjamins. 
Juanatey, Mayra (2020). Relaciones entre eventos y referencialidad en quichua santiagueño: de la gramática al discurso. Tesis doctoral no publicada. Universidad de Buenos Aires.

— (2019). Resumen y recapitulación discursiva en quechua: enlaces tail-head en quechua IIB y quechua IIC. LIAMES: Línguas Indígenas Americanas. Vol. 19, 125 https://doi.org/10.20396/liames.v19i1.8654493

— (2016). Revisión del sistema de conmutación de la referencia (switch-reference) del quichua santiagueño: Reducción sintáctica y especificación funcional de las construcciones en -s. RASAL Lingüística, 31-56.

Longacre, Robert E. (1985). Sentences as combinations of clauses. En: T. Shopen (ed.) Language typology and syntactic description. Volume 2: Complex Constructions. Cambridge University Press, 235-286.

Matthiessen, Christian M.I.M.; Thompson, Sandra A. (1988). The structure of discourse and "subordination". En: J. Haiman; S. A. Thompson (eds.) Clause Combining in Grammar and Discourse. (pp. 275-330). John Benjamins.

Overall, Simon (2009). The semantics of clause linking in Aguaruna. En: R.M.W. Dixon; A. Y. Aikhenvald (eds.) The Semantics of Clause Linking: A Cross-Linguistic Typology. (pp. 167-192). Oxford University Press.

Stirling, Lesley (1993). Switch-Reference and Discourse Representation. Cambridge University Press.

Tebes, Mario C.; Karlovich, Atila (2010). Sisa Pallana. Eudeba.

Tevez, Héctor. (2016). Uturungu upa. En: L. Albarracín (comp.) La quichua. Gramática, ejercicios y selección de textos, Vol. 3. Dunken.

Thompson, Sandra A. (1987). Subordination and narrative event structure. En: R. Tomlin (ed.) Coherence and grounding in discourse. (pp. 435-454). John Benjamins.

Thompson, Sandra A.; Longacre, Robert E. (1985). Adverbial clauses. En: T. Shopen (ed.) Language typology and syntactic description. Volume 2: Complex constructions. (pp. 171-234). Cambridge University Press.

Thompson, Sandra A.; Longacre, Robert E.; Hwang, Shin J. J. (2007). Adverbial clauses. En: T. Shopen (ed.) Language typology and syntactic description. Volume 2: Complex constructions. (pp. 237-269). Cambridge University Press.

Van Gijn, Rik (2016). Switch reference in Western South America. En: R. van Gijn; J. Hammond (eds.) Typological Studies in Language. (pp. 153-206). John Benjamins.

— (2014). "Repeated dependent clauses in Yurakaré". En: J. Hammond; D. Matić; S. van Putten; V. Galucio (eds.) Information Structure and Reference Tracking in Complex Sentences. (pp. 291-308). John Benjamins. 
Construcciones de enlace tail-head en quichua santiagueño

Van Valin, Robert D. (2005). Exploring the Syntax-Semantics Interface. Cambridge University Press. 